\title{
Corela
}

Cognition, représentation, langage

HS-32 | 2020

Les postures énonciatives

\section{Une posture énonciative peut en cacher une autre : rhétoriques de l'allusion et de la disposition dans les Pensées de Pascal}

\section{Laurent Susini}

\section{OpenEdition}

Journals

Édition électronique

URL : https://journals.openedition.org/corela/12137

DOI : $10.4000 /$ corela. 12137

ISSN : 1638-573X

Éditeur

Cercle linguistique du Centre et de I'Ouest - CerLICO

\section{Référence électronique}

Laurent Susini, « Une posture énonciative peut en cacher une autre : rhétoriques de l'allusion et de la disposition dans les Pensées de Pascal », Corela [En ligne], HS-32 | 2020, mis en ligne le 05 novembre 2020, consulté le 14 juillet 2021. URL : http://journals.openedition.org/corela/12137 ; DOI : https:// doi.org/10.4000/corela.12137

Ce document a été généré automatiquement le 14 juillet 2021.

\section{c.) (i) (2)}

Corela - cognition, représentation, langage est mis à disposition selon les termes de la licence Creative Commons Attribution - Pas d'Utilisation Commerciale - Partage dans les Mêmes Conditions 4.0 International. 


\title{
Une posture énonciative peut en cacher une autre : rhétoriques de l'allusion et de la disposition dans les Pensées de Pascal
}

\author{
Laurent Susini
}

\section{Les différents ordres de vérité dans les Pensées}

1 S'agissant des postures de la topique énonciative, Alain Rabatel a régulièrement insisté sur les deux points suivants :

2 1. Ces postures reposent sur «la déliaison de l'énonciateur et du locuteur » (Rabatel 2009 : 262).

3 2. Elles se définissent par « la position qu'adopte un énonciateur dans l'expression d'un PDV» (Rabatel $2007: 89$ ).

4 S'ils valent d'être à nouveau rappelés ici, c'est que l'un et l'autre semblent expliquer que les Pensées de Pascal puissent présenter un corpus a priori si privilégié pour l'étude des postures énonciatives - un corpus tout particulièrement propre à pousser la réflexion sur leurs instruments d'analyse dans certains de leurs retranchements.

5 Soit, tout d'abord, le premier point, concernant la déliaison du locuteur et de l'énonciateur. On posera d'emblée que les Pensées en théorisent le passage à la limite, en présentant cette déliaison non seulement comme le principe de toute énonciation, mais, plus profondément, comme la conséquence d'un étrange impératif de déliaison entre parole et pensée : selon les termes du fragment 645 , « II faut [...] dire comme les autres, mais ne pas penser comme eux ", et réserver dès lors, dans la béance creusée par ce mais, l'espace ambivalent de ce que Pascal appelle une "pensée de derrière ». Pensée de derrière d'ailleurs le plus souvent mal comprise, semblant participer d'une volonté de dissimulation, et ne se tenant pourtant derrière les mots voués à l'occulter, 
que parce que les mots échouent toujours à spécifier quelle vérité ils expriment. Ce qu'il convient sans doute d'éclaircir avant d'entrer plus avant dans le propos de cette étude.

6 Un simple exemple pourra servir de point de départ : la dynamique de renversement du pour au contre, selon les termes pascaliens, telle qu'on peut l'observer dans la gradation des différentes réponses apportées à la question du respect dû aux personnes de grande naissance.

Le peuple honore les personnes de grande naissance. Les demi-habiles les méprisent, disant que la naissance n'est pas un avantage de la personne, mais du hasard. Les habiles les honorent, non par la pensée du peuple, mais par la pensée de derrière. Les dévots, qui ont plus de zèle que de science, les méprisent, malgré cette considération qui les fait honorer par les habiles, parce qu'ils en jugent par une nouvelle lumière que la piété leur donne. Mais les chrétiens parfaits les honorent par une autre lumière supérieure. (Pensées, S. 124.)

7 La question ici débattue (Doit-on respecter les personnes de grande naissance?) reçoit deux sortes de réponses, partageant l'humanité en deux clans: d'un côté, le peuple, les habiles et les chrétiens parfaits, qui tiennent qu'il faut honorer les personnes de grande naissance; d'un autre côté, les demi-habiles et les dévots, qui tiennent qu'il ne faut pas honorer les personnes de grande naissance. À deux propositions logiquement contradictoires (il faut honorer les personnes de grande naissance vs il ne faut pas honorer les personnes de grande naissance) répondent ainsi cinq énoncés : cinq énoncés pour certains formellement identiques quoique tous différents; et cinq énoncés par ailleurs tous vrais en leur ordre bien qu'inégalement vrais, c'est-à-dire, de plus en plus vrais au fur et à mesure que le fragment progresse. La pensée de derrière s'enracine en ce double point : dans ce flottement et dans cette gradation, avec deux conséquences corollaires.

8 1. La première est que s'accordant, par exemple, pour dire qu'il faut honorer les personnes de grande naissance, le peuple, les habiles et les chrétiens parfaits pourront donner l'illusion de co-énoncer la même position, alors que les habiles sur-énonceront dans les faits ce que dira le peuple, et les chrétiens parfaits à leur tour ce que surénonceront les habiles. En clair, une posture énonciative peut en cacher une autre...

9 2. ... en sorte qu'on ne saurait donc définir la position qu'adopte un énonciateur dans l'expression d'un PDV sans comprendre à la lettre cette notion de point de vue, à savoir comme site perspectif précis à partir duquel justifier d'une certaine façon de voir et de penser: où l'on retrouve le second point rappelé par Alain Rabatel concernant les postures énonciatives. C'est qu' «il faut avoir un point fixe pour [...] juger du langage » (Pensées, S. 576), et qu'une posture énonciative ne saurait donc être, semble-t-il, assignable sur la base, en tant que telle infiniment plastique, d'un simple contenu propositionnel, grammatical, syntaxique ou sémantique. Son assignation s'impose comme le fruit d'un ensemble de calculs pragmatiques susceptibles de brouiller les limites entre "description » et « interprétation», en impliquant avant tout de savoir, ou de déterminer, qui parle, c'est-à-dire plus exactement d'où parle celui qui parle, dès lors qu'il importe ainsi de conjurer la forme d'occultation, contrainte par le langage, du point de vue depuis lequel s'exprime tout énoncé donné.

Or telle est bien la grande difficulté à laquelle confrontent les Pensées : ce point de vue est bien souvent inassignable en tant que tel, et il n'en va pas ici que du caractère fragmentaire du recueil, multipliant par nature des "phrases sans texte ${ }^{1}$ ». Dénué de tout contexte, il va de soi qu'un fragment comme «Le silence éternel de ces espaces infinis m'effraie.» (Pensées, S. 233) puisse se prêter à de multiples interprétations, selon 
qu'on le considère énoncé du point de vue d'un athée, du point de vue d'un chrétien, ou du point de vue de la figure d'apologiste mise en scène dans les Pensées. Et ne serait-ce d'ailleurs que dans ce dernier cas, la difficulté ne serait pas plus dénouée, car il serait alors aussi envisageable de défendre du fragment une lecture en termes de coénonciation empathique avec le lectorat visé (nous, les adorateurs du dieu caché, partageons les mêmes affres que vous qui êtes athées), ou une lecture, à l'inverse, en termes de sur-énonciation polémique (entendez, vous athées, la manière dont vous parlez, et dans quels affres insupportables vous plonge votre incroyance). En l'absence de cadre enchâssant, et donc de scène énonciative clairement définie, cette indécision n'a rien d'étonnant: toute proposition contient en germes une infinité d'énoncés éventuellement contradictoires, et l'on ne saurait donc marquer de posture énonciative stable en l'absence d'un PDV bien défini, autant dire, en présence de tous les PDV virtuellement possibles.

11 Ce sur quoi cette étude voudrait cependant attirer l'attention, ce sont ces cas autrement singuliers (et n'ayant rien à voir avec le problème bien particulier posé par les phrases sans textes), où, dans les Pensées, l'apparence d'une posture énonciative vient insidieusement à en recouvrir une autre : ces cas où, brouillant à dessein les frontières des PDV en présence, Pascal tend à superposer de manière insinuante différentes postures énonciatives, en investissant sur le mode d'une ironie sérieuse la zone de flou ménagée par diverses propositions cristallisant potentiellement divers points de vue diversement vrais - ce qui lui permet ainsi de faire siennes, en les convertissant, les armes diaboliques de l'Insinuateur, ou, comme on voudra, de l'imposteur, créature par définition sans posture, et ne devant ainsi son «charme » qu'à cette « indétermination ${ }^{2}$ » qui lui permet de s'adapter à toutes les situations, dans « la disparition de toutes positions ${ }^{3} »$ fixes.

Il s'agira d'interroger ici ces phénomènes de superposition et de faux-semblants énonciatifs sous deux angles conjoints :

1. d'abord sous l'angle des tensions entre éthos discursif et posture énonciative ;

14 2. ensuite - ce qui n'a été que rarement étudié - sous l'angle des incidences de la dispositio sur la requalification des postures énonciatives à l'œuvre.

\section{La tension entre éthos discursif et posture énonciative}

Concernant le premier point, il convient avant tout de rappeler que, pour une bonne part, les Pensées circonscrivent le chantier d'une forme de discours de défense du christianisme visant la conversion d'un public d'honnêtes gens tièdes ou incroyants, avec ce que le terme d'honnêteté implique, au XvII siècle, de prétention à l'universalité, de souriante politesse des mœurs et d'euphorie conversationnelle. À ce titre, l'une des grandes pistes rhétoriques suivies par Pascal est la suivante: en s'autorisant de l'héritage salésien, qui pense la possible continuité des sphères du monde et de la dévotion, et en s'appuyant plus généralement sur une pensée de l'éclatement et de la gradation du vrai (celle-là même qu'est contrainte d'investir la pensée de derrière), l'auteur des Pensées s'emploie bien souvent, sur le mode de la sourde consonance, à recouvrir d'un éthos d'honnêteté le caractère chrétien de sa perspective, et à ménager ainsi une illusion de connivence avec son lecteur en s'employant à co-énoncer en 
honnête homme - avec tout ce que cela suppose d'ostentation dans le partage de valeurs et de discours communs - ce qu'il assume et sur-énonce en réalité du point de vue de sa foi, c'est-à-dire non d'un point de vue contraire, mais pour autant à un tout autre niveau.

À cet égard, tel qu'il se déploie dans un grand nombre de fragments, l'éthos d'honnête homme construit par l'auteur des Pensées revêt, semble-t-il, une double fonction :

- la fonction de séduire la figure de lecteur construite par le texte, en surmarquant les traits les plus symptomatiques d'une posture de co-énonciation appelée à orchestrer et à souligner la convergence supposée des PDV de Pascal et de son lecteur ;

18 - mais aussi bien la fonction de voiler, en recouvrant, estompant ou effaçant réciproquement la plupart des marques d'une posture de sur-énonciation, appelée à réverbérer, sur le mode de l'allusion insinuante, la nature du PDV surplombant depuis lequel l'apologiste du christianisme s'autorise du masque de l'honnêteté.

De cette tension structurante, le fragment suivant présente une illustration exemplaire :

Honnête homme.

Il faut qu'on n'en puisse [dire] ni : il est mathématicien, ni prédicateur, ni éloquent, mais il est honnête homme. Cette qualité universelle me plaît seule. Quand en voyant un homme on se souvient de son livre, c'est mauvais signe. Je voudrais qu'on ne s'aperçût d'aucune qualité que par la rencontre et l'occasion d'en user, ne quid nimis, de peur qu'une qualité ne l'emporte, et ne fasse baptiser. Qu'on ne songe point qu'il parle bien, sinon quand il s'agit de bien parler. Mais qu'on y songe alors. (Pensées, S. 532)

20 S'y donne à lire toute l'attention de Pascal à interroger les qualités de l'honnête éloquence pour les réfléchir à son tour, et pour se composer ce faisant un éthos d'honnêteté propre à lui permettre de se faire l'apparent co-énonciateur d'un PDV d'honnête homme qu'il sur-énonce dans les faits.

Ici, cette tension énonciative est d'emblée perceptible dans la distribution des pronoms personnels sujet je et $i l$, qui inscrivent le locuteur dans une position toute paradoxale d'intériorité et d'extériorité par rapport à son objet. Le je ne dit pas qu'il est honnête homme, il parle de l'honnête homme, mais de manière à faire comprendre qu'il en est un lui-même - et ce dispositif emblématise discrètement l'ambivalence énonciative du fragment. Certes, la nature des valeurs revendiquées par la P1 (honnêteté, universalité, modération en tout, éloquence naturelle), la mention explicite de la maxime delphique latinisée par Térence, ce ne quid nimis ${ }^{4}$, « rien de trop », qui figure le cœur de la pensée gréco-latine réappropriée par l'homme de cour à la suite de Montaigne, l'apparence de spontanéité sans apprêt entretenue par la dislocation de la syntaxe ( Quand..., c'est... »), la fine raillerie des métaphores («baptiser ») et des équivoques («mauvais signe », s'agissant d'un homme-livre), le retournement malicieux de la pointe finale aux accents de litote ("Mais qu'on y songe alors») - tout, dans ce fragment, concourt à la mise en scène d'un éthos d'honnêteté susceptible d'attirer la confiance du lecteur visé par Pascal. Mais à l'évidence, la mise en scène de cet éthos recouvre, outre des enjeux strictement rhétoriques, des enjeux apologétiques. Prétendre ne se plaire qu'à l' " univers[alité] ", c'est, au-delà de tout ce qui touche à l'honnêteté, baliser la voie, ne serait-ce qu'étymologiquement, vers la religion catholique. Et revendiquer de la même manière la maxime delphique du rien de trop, c'est souterrainement disposer à ressentir, le moment venu, la nécessité de cette «temp[érance]» de «la crainte avec 
l'espérance " (Pensées, S. 240) que ménagent les "contrepoids» (Pensées, S. 383) du christianisme. À quoi s'ajoutent enfin sur le mode de la connotation les inflexions ou résonances chrétiennes des termes utilisés - baptiser au premier chef, bien sûr, mais également occasion qui appartient à ce vocabulaire de la théologie morale que n'ont cessé d'interroger Les Provinciales, ou user qui relève du vocabulaire technique de la théologie augustinienne : "Jouir d'une chose, c'est s'attacher par amour à une chose, pour elle-même. User de quelque chose, c'est en revanche rapporter ce dont on a l'usage à l'obtention de ce que l'on aime, si toutefois il faut l'aimer ${ }^{5}$ ». Autant d'indices convergents, et manifestant donc obliquement, sur le mode de l'allusion, ce PDV second, catholique pour sa part, depuis lequel se trouve sur-énoncé l'ensemble du fragment - et donc, au premier chef, le PDV co-énoncé de l'honnête homme, au principe de ce qu'on pourrait appeler un trope topique, ou postural, recouvrant sous un sens littéral explicitement co-énoncé un sens figuré allusivement sur-énoncé.

Et c'est ainsi que l'éthos d'honnêteté construit par le fragment agit finalement comme une forme de trompe-l'œil sans réelle tromperie - ou plus exactement, comme le principe d'une dissimulation sans simulation. Il recouvre le PDV d'un énonciateur catholique, mais sans pour autant participer d'une feinte adhésion au PDV asserté de l'énonciateur honnête homme, qu'il dépasse en l'intégrant. Le je qui l'instancie ne feint pas d'adhérer aux valeurs de l'honnêteté - «[1]e chrétien », selon Pascal, « est l'honnête homme achevé et non pas la négation de l'honnête homme ${ }^{6} »-$, il feint plus subtilement d'assumer en honnête homme les valeurs de l'honnêteté, alors qu'il les assume en catholique. En un mot, il semble ne multiplier les gages de co-énonciation que pour mieux recouvrir les marques de sa sur-énonciation. Et tel est donc l'éthos accommodant élaboré dans les Pensées, que les PDV co-énoncés qu'il s'ingénie à mettre en scène se voient sourdement, allusivement, sur-énoncés depuis un PDV chrétien, sur le mode d'une tension polyphonique à peine perceptible et d'une ironie sans véritable grincement.

En somme, ce que donne à lire un tel fragment, c'est le possible travail de recouvrement d'une posture énonciative (de sur-énonciation) par une autre (de coénonciation) ou, pour le formuler autrement, une entreprise de "dissimul[ation]» énonciative "par assimilation ${ }^{7}$ ", attentive à ne pas trahir le fait qu'on se contente de citer ce qu'on veille à donner l'illusion de dire en son propre nom.

\section{La réécriture de Montaigne : un art de la dispositio imitant la rhétorique biblique}

D'où l'intérêt a contrario d'étudier dans un second temps quelques-uns de ces moments où, loin de dissimuler qu'il les cite, le Pascal des Pensées vient au contraire à réécrire expressément, sinon à plagier tout à fait, les Essais de Montaigne, bréviaire lu et relu de l'honnête homme du milieu du XVII siècle. L'intention du procédé est, à première vue, transparente: à travers l'entreprise de réécriture des Essais, il s'agit, selon toute vraisemblance, d'éveiller en l'honnête lecteur un sentiment de familiarité et de reconnaissance immédiate, et plus encore de l'inviter à une lecture idéalement connivente, en prise directe avec l'apparente co-énonciation d'une doxa commune. Mais, là encore, une telle co-énonciation n'est jamais qu'apparente, dans la mesure, cette fois, où la manière qu'à Pascal de redistribuer, c'est-à-dire de recomposer, la matière réécrite des Essais tend alors à se faire l'indice concomitant d'une posture 
énonciative seconde de sur-énonciation, marquant dans le même mouvement l'intégration et le dépassement de la première.

C'est ce qui se comprendra aisément à l'aide d'un simple exemple, la réécriture des Essais auquel se livre le bref fragment 87 :

Pascal - Inconstance. / Les choses ont diverses qualités et l'âme diverses inclinations, car rien n'est simple de ce qui s'offre à l'âme, et l'âme ne s'offre jamais simple à aucun sujet. De là vient qu'on pleure et qu'on rit d'une même chose. (Pensées, S. 87)

Montaigne - il faut considerer, comme nos ames se trouvent souvent agitees de diverses passions. [...] D'où nous voyons non seulement les enfans, qui vont tout naifvement apres la nature, pleurer et rire souvent de mesme chose [...]. Nulle qualité nous embrasse purement et universellement. [...] car chasque chose a plusieurs biais et plusieurs lustres.(Les Essais, I, 37, p. 233-234)

De l'hypotexte des Essais à l'hypertexte des Pensées, la relation de dérivation ne fait aucun doute, et la reprise des mêmes arguments s'accompagne d'ailleurs de l'emprunt d'une grande part du matériel lexical. Seule change vraiment, d'un texte à l'autre, la disposition de la matière. De fait, là où Montaigne évoquait

1. l'âme agitée de diverses passions

2. la capacité des enfants à rire et pleurer d'une même chose,

3. les divers biais de chaque chose,

Pascal, pour sa part, mentionne d'abord, au titre de causes, les diverses qualités de chaque chose et les diverses inclinations de l'âme, puis au titre de conséquence, la capacité de l'homme à rire et à pleurer d'une même chose - soit, dans l'ordre montaignien, les points 3), 1), 2). Ce qu'on pourra interpréter de deux manières conjointes: d'abord comme une façon de conjurer la "confusion de Montaigne », en séparant les causes de leurs conséquences; mais aussi bien, comme un moyen, sans doute, de régler la réécriture des Essais sur les modes de composition les plus éprouvés de la rhétorique biblique - ce qui appelle, en ce point, d'ouvrir une brève parenthèse.

31 Car qu'est-ce, exactement, que la rhétorique biblique ? Pour le dire en un mot, c'est une rhétorique procédant à tout instant, sur les plans micro- et macro-structurel, par mouvements symétriques: ce qui pourrait sembler en somme assez commun, si ces mêmes symétries ne valaient avant tout principe de composition, et, au-delà d'une simple tension poétique, invitation à saisir, par la spécificité de leur distribution, la structure et les articulations du discours. Du plus petit au plus grand niveau, en effet, tous les textes bibliques étudiés jusqu'ici dans le sillage des travaux fondateurs de Roland Meynet ont manifesté une même tendance à procéder, non de manière linéaire, de $A$ vers $B$ puis de $B$ vers $C$, mais, suivant des règles très précises, et constamment vérifiées, par enchâssements successifs de structures parallèles, le plus souvent organisées autour d'un centre et repérables sur la base de réseaux de symétries partielles. Ce que rend très sensible ce qu'on appelle leur réécriture, c'est-à-dire la manifestation de leur composition par les simples modalités, très réglées elles aussi, de leur mise en page ${ }^{8}$.

32 Qu'on prenne, pour plus de clarté, l'exemple de ce bref passage de l'évangile de Marc (15,21-24), ici traduit mot à mot du grec :

${ }^{21} \mathrm{Et}$ ils requièrent un qui passait, Simon de Cyrène, qui revenait des champs, le père d'Alexandre et de Rufus, afin qu'il prenne sa croix. ${ }^{22} \mathrm{Et}$ ils le portent au lieu Golgotha, ce qui est traduit le lieu du Crâne, ${ }^{23}$ et ils lui donnaient du vin mêlé de myrrhe, mais il n'en prit pas. ${ }^{24} \mathrm{Et}$ ils crucifient lui. 
et assorti de la récriture qu'en a proposée Roland Meynet $^{9}$ (fig. 1) :

\begin{tabular}{|c|c|c|}
\hline$+{ }^{21} \mathbf{E t}$ ils requièrent & un qui passait, & \\
\hline : simon & de cyrène, & \\
\hline $\begin{array}{c}+ \text { qui revenait } \\
\text { : le père }\end{array}$ & $\begin{array}{l}\text { des champs, } \\
\text { d'Alexandre }\end{array}$ & et de Rufus, \\
\hline$=$ afin qu'il & PRENNE & SA CROIX. \\
\hline $\begin{array}{l}+{ }^{22} \mathbf{E t} \text { ils le portent } \\
\text { : ce qui est traduit }\end{array}$ & $\begin{array}{l}\text { au lieu } \\
\text { lien }\end{array}$ & $\begin{array}{l}\text { Golgotha, } \\
\text { du crâne, }\end{array}$ \\
\hline $\begin{array}{c}+{ }^{23} \text { et ils lui donnaient } \\
\text { : mais lui }\end{array}$ & $\begin{array}{l}\text { du vin } \\
\text { n'(en) PRIT pas. }\end{array}$ & mêlé-de-myrrhe, \\
\hline$={ }^{24} \mathbf{E t}$ ILS CRUCIFIENT & lui. & \\
\hline
\end{tabular}

Fig. 1 - Récriture de la partie Mc 15,21-24

Cette dernière rend immédiatement sensibles deux phénomènes conjoints :

1. d'une part, les symétries partielles qui organisent la matière textuelle des quatre versets :

1.1. même usage transversal du coordonnant $e t$;

1.2.même focalisation sur la croix dans les segments finaux ;

1.3. et dans les segments initiaux, même mouvement réglant l'usage des noms propres, de leur simple mention («Simon», «Golgotha»), à leur explicitation (" père d'Alexandre et de Rufus », « le Crâne »);

2. et, d'autre part la manière dont ces mêmes symétries partielles assurent le parallélisme global des deux unités textuelles réparties de part et d'autre de la ligne en pointillés, et, plus avant, leur réunion dans l'unité textuelle supérieure de ce qu'on appellera une partie, de structure ici parallèle.

Mais, ce qui est plus singulier encore - et qui engage précisément la spécificité de la rhétorique biblique -, le fait est que ces mêmes phénomènes demeurent toujours observables aux niveaux textuels supérieurs, de sorte qu'en remontant progressivement de l'un à l'autre, il devient également possible de suivre, au fil des récritures successives reproduites par les figures 2,3 et 4 , les modalités de la participation de cette même partie Mc 15,21-24 à un passage de structure concentrique (fig. 2) : 


\begin{tabular}{|c|c|c|}
\hline $\begin{array}{c}+{ }^{21} \text { Et ils requièrent } \\
: \text { Simon }\end{array}$ & $\begin{array}{l}\text { un qui passait, } \\
\text { de Cyrène, }\end{array}$ & \\
\hline $\begin{array}{l}\text { + qui revenait } \\
\text { : le père }\end{array}$ & $\begin{array}{l}\text { des champs, } \\
\text { d'Alexandre }\end{array}$ & et de Rufus, \\
\hline$=$ afin qu'il & PRENNE & SA CROIX. \\
\hline $\begin{array}{l}+{ }^{22} \text { Et ils le portent } \\
\text { : ce qui est traduit }\end{array}$ & $\begin{array}{l}\text { au lieu } \\
\text { lieu }\end{array}$ & $\begin{array}{l}\text { Golgotha, } \\
\text { du Crâne, }\end{array}$ \\
\hline $\begin{array}{c}+{ }^{23} \text { et ils lui donnaient } \\
\text { : mais lui }\end{array}$ & $\begin{array}{l}\text { du vin } \\
\mathrm{n}^{\prime}(\mathrm{en}) \text { prit pas. }\end{array}$ & mêlé-de-myrrhe, \\
\hline$={ }^{24} \mathrm{Et}$ ILS CRUCIFIENT & lui. & \\
\hline
\end{tabular}

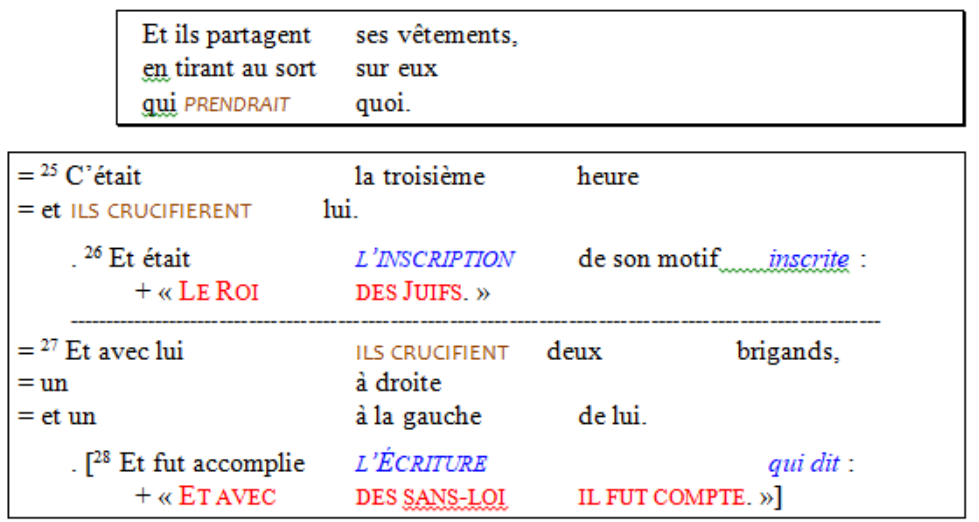

Fig. 2 - Récriture du passage Mc 15,21-28

puis à une sous-séquence de structure parallèle (fig. 3) :

${ }^{21}$ Et ils requièrent un passant, Simon de Cyrène, qui venait des champs, le père d'Alexandre et de Rufus, afin qu'il prît sa CROLX.

${ }^{22}$ Et ils le portent au lieu Golgotha ce qui se traduit lieu du Crâne, ${ }^{23}$ et ils lui donnaient du vin mêlé de myrrhe, mais il n'en prit pas, ${ }^{24}$ et ils le crucifient.

Alors ils partagent ses vêtements, en les tirant au sort pour savoir qui prendrait quoi.

${ }^{25}$ C'était la troisième heure quand ils le crucifièrent. ${ }^{26}$ L'inscription de sa condamnation était ainsi inscrite : "LE ROI DES JUIFS. "

${ }^{27}$ Et avec lui ils crucifient deux brigands, un à droite et un à gauche.

[ ${ }^{28}$ Et fut accomplie l'Écriture qui dit : «Et avec des sans-loi il fut compté. »]

${ }^{29}$ Les passants l'injuriaient, hochant la tête et disant : « Hé ! Toi qui détruis le sanctuaire et le rebâtis en trois jours, ${ }^{30}$ sauve-toi toi-même, descendant de la CROLX ! »

${ }^{31}$ Pareillement aussi les grands prêtres, se jouant entre eux avec les scribes, disaient : "Il en a sauvé d'autres, il ne peut se sauver lui-même! ${ }^{32}$ Le Christ, LE ROI D'ISRAËL, qu'il descende maintenant de la CROLX, afin que nous voyions et que nous croyions ! »

Aussi cenx qui avaient été crucifiés avec lui l'insultaient.

Fig. 3 - Récriture de la sous-séquence Mc 15,21-32

puis à une séquence de structure concentrique (fig. 4), 


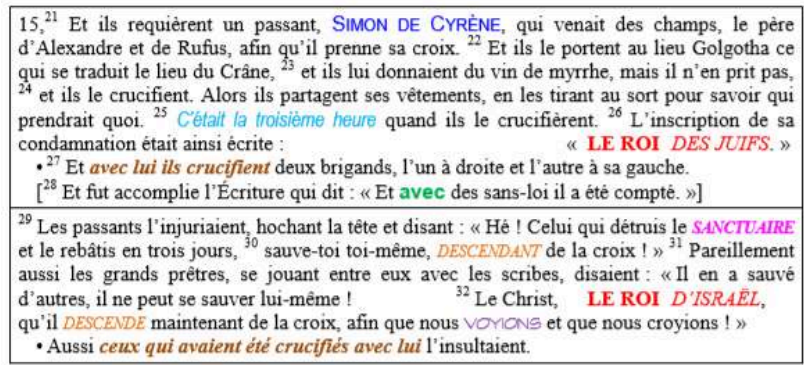

-Aussi ceux qui avaient été crucifiés avec lui l' insultaient.

${ }^{33}$ Quand arriva la sixième heure, une ténèbre arriva sur la terre toute-entière jusqu'à la neuvième heure. ${ }^{34} \mathrm{Et}$ à la neuvième heure, Jésus clama à GRAND CRI : «Élồi, Élôî, lema sabachtani? » ce qui se traduit : «Mon DIEU, mon DIEU, pourquoi m'as-tu abandonné ? 》

Certains de ceux qui se tenaient-là ayant entendu dirent: "Voici qu'il appelle ELIE ! " ${ }^{36}$ Certain ayant couru, ayant rempli une éponge de vinaigre, l'ayant fixée à un roseau, l'abreuvait en disant : « Laissez ! VOYONS si ÉLIE vient le DESCENDRE! ”

\begin{tabular}{|c|}
\hline 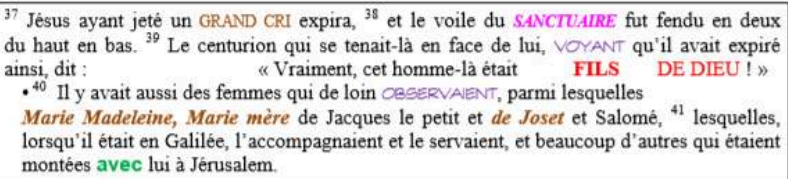 \\
\hline 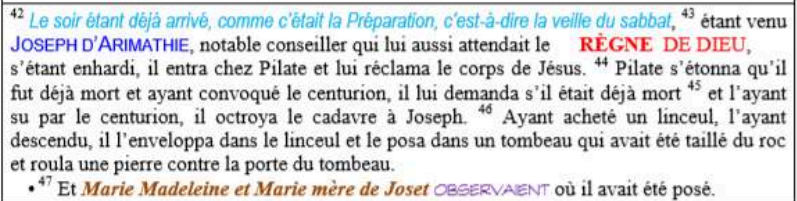 \\
\hline
\end{tabular}

Fig. 4 - Récriture de la séquence Mc 15,21-47

... et ainsi de suite, jusqu'à remonter potentiellement, à force d'intégrations successives, à la structure de l'évangile entier.

Or l'évangile de Marc n'est sans doute pas l'objet de cette étude. Mais une fois rappelée la singularité des principes de composition qui l'informent, au même titre que les autres livres de la Bible, qu'on s'essaie à récrire le fragment S. 87 (fig. 5) :

\begin{tabular}{|llll|}
\hline $\begin{array}{l}\text { Les choses } \\
\text { et l'âme }\end{array}$ & $\begin{array}{l}\text { ont } \\
\text { diverses }\end{array}$ & $\begin{array}{l}\text { diverses } \\
\text { inclinations }\end{array}$ & qualités \\
\hline car & $\begin{array}{l}\text { rien } \\
\text { de ce qui }\end{array}$ & $\begin{array}{l}\text { n'est SIMPLE } \\
\text { à l'âme }\end{array}$ & \\
et l'âme & ne s'offre & jamais & SIMPLE \\
à aucun & sujet & & \\
- de là $_{\text {d'une même }}$ & vient & qu'on pleure & et qu'on rit \\
\hline
\end{tabular}

Fig. 5 - Récriture du fragment S. 87

Il apparaîtra alors le fait suivant: la réorganisation de l'hypotexte montaignien à laquelle procède ce fragment y participe conjointement de l'élaboration d'une composition des plus réminiscentes de la rhétorique biblique, prenant ici la forme d'une unité textuelle tripartite à structure concentrique, clairement marquée par le parallélisme de ses segments-cadre et par le parallélisme de ses segments enchâssés. 
41 Et le phénomène n'est naturellement pas sans incidence. Réécrire Montaigne en pastichant les Écritures, c'est naturellement modifier le sens de la matière réécrite, dès lors que c'est faire parler la nature humaine du point de vue de la surnature, en marquant indirectement, l'intégration et le dépassement de la première par la seconde. En sorte que, par le biais, cette fois, de la seule disposition, c'est donc, à nouveau, surénoncer sourdement en chrétien ce qu'on semble co-énoncer explicitement en honnête homme.

42 Où l'on pourra être tenté de voir l'effet d'une reconstruction hasardeuse, voire d'une surinterprétation délirante.

43 Mais que l'on prenne alors, entre autres exemples, le fragment S. 226, dérivé lui aussi d'un passage des Essais :

Pascal - La prévention induisant en erreur. / C'est une chose déplorable de voir tous les hommes ne délibérer que des moyens et point de la fin. Chacun songe comment il s'acquittera de sa condition, mais pour le choix de la condition, et de la patrie, le sort nous le donne. / C'est une chose pitoyable de voir tant de Turcs, d'hérétiques, d'infidèles, suivre le train de leurs pères, par cette seule raison qu'ils ont été prévenus chacun que c'est le meilleur. Et c'est ce qui détermine chacun à chaque condition, de serrurier, soldat, etc. / C'est par là que les sauvages n'ont que faire de la Provence. (S. 226)

Montaigne - Le principal effet de sa puissance [celle de la coutume] c'est de nous saisir et empiéter de telle sorte, qu'à peine soit-il en nous, de nous ravoir de sa prise, et de rentrer en nous, pour discourir et raisonner de ses ordonnances. De vrai, parce que nous les humons avec le lait de notre naissance, et que le visage du monde se présente en cet état à notre première vue, il semble que nous soyons nés à la condition de suivre ce train. Et les communes imaginations, que nous trouvons en crédit autour de nous, et infuses en notre âme par la semence de nos pères, il semble que ce soient les générales et naturelles. C'est par l'entremise de la coutume que chacun est content du lieu où la nature l'a planté et les sauvages d'Écosse n'ont que faire de la Touraine, ni les Scythes de la Thessalie. (Les Essais, I 23, op. cit., p. 115-116)

D'un texte à l'autre, une nouvelle fois, la relation de dérivation hypertextuelle n'est guère discutable. Seule dissonance étrange, cependant, au milieu de constants jeux d'échos lexicaux, là où Montaigne convoquait pour finir «la Touraine » et «la Thessalie ", Pascal préfère, pour sa part, évoquer «la Provence». Or, certes, la Provence était à la mode à ce moment du XVII ${ }^{\mathrm{e}}$ siècle ${ }^{10}$. Mais le sens de cette substitution, par ailleurs si singulière, tend à s'éclairer davantage, une fois exposée à la lumière de l'hypothèse risquée il y a un instant: Pascal, réécrivant Montaigne, pastiche aussi les Écritures.

C'est ce que manifeste la récriture du fragment (fig. 6). 


\begin{tabular}{|c|c|c|c|}
\hline La prévention & induisant & en erreur. | & \\
\hline $\begin{array}{l}\text { c'est } \\
\text { de voir } \\
\text { ne délibérer }\end{array}$ & $\begin{array}{l}\text { une chose } \\
\text { tous les hommes } \\
\text { que des moyens }\end{array}$ & $\begin{array}{l}\text { déplorable } \\
\text { et point }\end{array}$ & de la fin. \\
\hline $\begin{array}{l}\text { CHACUN } \\
\text { comment }\end{array}$ & $\begin{array}{l}\text { songe } \\
\text { il s'acquittera }\end{array}$ & de sa condition, & \\
\hline $\begin{array}{l}\text { mais } \\
\text { le sort }\end{array}$ & $\begin{array}{l}\text { pour le } C H O I X \\
\text { nous }\end{array}$ & $\begin{array}{l}\text { de la condition, } \\
\text { le donne. }\end{array}$ & et de la PATRIE, \\
\hline $\begin{array}{l}\text { C'est } \\
\text { de voir } \\
\text { suivre }\end{array}$ & $\begin{array}{l}\text { une chose } \\
\text { tant de Turcs, } \\
\text { le train }\end{array}$ & $\begin{array}{l}\text { pitoyable } \\
\text { d'hérétiques, } \\
\text { de leurs PĖREs, }\end{array}$ & d'infidèles, \\
\hline $\begin{array}{l}\text { par } \\
\text { qu'ils ont été prévenus }\end{array}$ & $\begin{array}{l}\text { cette seule raison } \\
\text { CHACUN }\end{array}$ & que c'est & le meilleur. \\
\hline $\begin{array}{l}\text { Et c'est } \\
\text { à CHAQUE condition }\end{array}$ & $\begin{array}{l}\text { ce qui } \\
\text { t, de serrurier, }\end{array}$ & $\begin{array}{l}\text { DETERMINE } \\
\text { soldat, }\end{array}$ & $\begin{array}{l}\text { CHACUN } \\
\text { etc. }\end{array}$ \\
\hline $\begin{array}{l}\text { C'est } \\
\text { que les sauvages }\end{array}$ & $\begin{array}{l}\text { par là } \\
\text { n'ont que faire }\end{array}$ & de la Provence. & \\
\hline
\end{tabular}

Fig. 6 - Récriture du fragment S. 226

Elle le donne à reconsidérer sous la forme d'une unité textuelle à nouveau tripartite, de structure à nouveau concentrique, et clairement marquée, à nouveau, par le parallélisme de ses segments-cadre comme par celui de ses morceaux centraux.

Or à quoi tient précisément le parallélisme des segments-cadres ? En l'occurrence, à une simple réverbération du signifiant, cet écho de prévention ménagé par Provence. Reprendre à Montaigne la mention de la Thessalie ou de la Touraine, c'était s'interdire de ménager le parallélisme des morceaux cadres du passage. Mais recourir à la Provence, c'était, par le biais de la malicieuse paronomase prévention/Provence, s'assurer de refermer la boucle du fragment et de l'offrir ainsi à ce type de lecture circulaire virtuellement toujours recommencée - et de ce fait méditative -, expressément visé par le mode de composition si spécifique des Écritures.

Ce dont on pourrait faire valoir d'autres exemples convergents ${ }^{11}$, et qui aboutiraient aux deux mêmes conclusions, intéressant la démarche pascalienne pour la première, et la manifestation potentiellement émergentiste du point de vue pour la seconde.

1. Dans la perspective de Pascal, en effet, traduire dans les cadres de la rhétorique biblique « la façon simple, naturelle et ordinaire $»^{12}$ de Montaigne, c'est, par le biais de la simple disposition, se donner les moyens d'envelopper une posture énonciative dans une autre (avec ce que cet enveloppement suppose expressément de conservation) et de sur-énoncer donc du point de vue de la foi ce qu'on semble co-énoncer (et qu'on assume de fait dans son ordre) du point de vue de l'honnêteté.

2. Mais de ce fait, la posture enveloppante ne se manifeste pour l'essentiel qu'à travers une synergie de composantes textuelles, de niveaux organisationnels et de faits de style très hétérogènes, qui ne permettraient pas spécialement de la repérer, examinés 
isolément. D'où l'importance à cet égard du montage discursif garant de leur articulation, et qui les co-oriente sur le plan sémantique.

\section{Conclusion}

51

52

Cette étude se donnait un double objet :

- questionner les liens entre éthos discursif et posture énonciative - et inviter, ce faisant, à se garder de recouvrir une question par l'autre ;

53 la disposition dans l'élaboration de ce que j'ai proposé d'appeler des tropes topiques, ou posturaux.

54 In fine, l'essentiel de son propos semble pourtant s'être joué ailleurs - dans cette lumière inédite que permet de jeter sur le concept de posture sa mise à l'épreuve du mouvement dialectique à l'œuvre dans la logique et l'ontologie pascaliennes. Car c'est depuis un nouveau point de vue, sans doute, qu'est désormais susceptible d'être appréhendé l'un des apports les plus décisifs des propositions d'Alain Rabatel (voir 2007 : 94-95) en matière de topique énonciative : affranchir du binarisme réducteur d'une opposition accord/désaccord l'étude de PDV en co-construction.

\section{BIBLIOGRAPHIE}

Montaigne, Les Essais, éd. P. Villey, Paris, Quadrige / PUF, 1992.

Pascal, Pensées, opuscules et lettres, éd. P. Sellier et L. Plazenet, Paris, Classiques Garnier, coll. « Bibliothèque du XVII ${ }^{\mathrm{e}}$ siècle ", 2010.

Maingueneau Dominique, 2012, Phrases sans texte, Paris, Armand Colin.

Meynet Roland, 1999, Jésus Passe, Paris, Le Cerf.

Meynet Roland, 2007, Traité de rhétorique biblique, Paris, Lethielleux.

Rabatel Alain, 2007, « Les enjeux des postures énonciatives et de leur utilisation en didactique », Éducation et didactique, vol. 1, $\mathrm{n}^{\circ}$ 2, Presses Universitaires de Rennes.

Rabatel Alain, 2009, Homo Narrans. Pour une analyse énonciative et interactionnelle du récit, vol. 1, Limoges, Lambert-Lucas.

Requemora-Gros Sylvie (dir.), 2017. Voyages, échanges, rencontres au XVII siècle, Tübingen, Narr Verlag, collection "Biblio 17".

Susini Laurent, 2011, « Pascal, Montaigne et la Bible. Un faux pastiche peut en cacher un vrai », Littératures Classiques, n 74, p. 91-106. 


\section{NOTES}

1. On emprunte ici le concept développé par Dominique Maingueneau (2012).

2. Herman Parret, Le Sublime du quotidien, Paris-Amsterdam, Éditions Hadès-Benjamins, 1988, p. 87.

3. Alain Arnaud, «Évanescences », Traverses, $\mathrm{n}^{\circ}$ 18, 1980, p. 91.

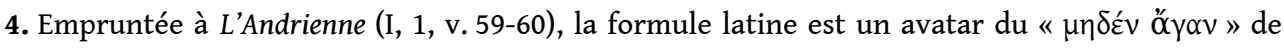
Solon.

5. Saint Augustin, Enseigner le christianisme, I, IV, 4, dans Euvres, vol. 3, Paris, Gallimard, « Bibliothèque de la Pléiade ", 2002, p. 11.

6. G. Ferreyrolles, suite à la communication de L. Thirouin, « Pascal et le savoir-vivre (esthétique de la vie sociale) », dans Pascal-Port-Royal-Orient-Occident. Actes du colloque de l'Université de Tokyo, 27-29 septembre 1988, dir. T. Goyet, Paris, Klincksieck, 1991, p. 280.

7. L. Marin, «Secret, dissimulation et art de persuader chez Pascal », Pascal et Port-Royal, Paris, PUF, 1997, p. 96.

8. Voir sur tous ces points Roland Meynet, 2007.

9. Jésus Passe, Cerf, 1999, p. 303-330.

10. Voir notamment Sylvie Requemora-Gros (dir.), 2017.

11. Voir Laurent Susini (2011).

12. Les Essais, « Au lecteur », op. cit., p. 3.

\section{RÉSUMÉS}

La pensée de l'éclatement du vrai défendue par Pascal autorise l'apologiste des Pensées à coénoncer en honnête homme ce qu'il sur-énonce en chrétien. En faisant valoir le jour nouveau jeté par la «topique énonciative» sur le mouvement dialectique à l'œuvre dans la logique et l'ontologie pascaliennes, on montre tout particulièrement la part des rhétoriques de l'allusion et de la disposition dans l'élaboration de ce qu'on propose d'appeler des « tropes posturaux ».

As a thinker of a fragmented thruth, the apologist of the Pensées co-enunciates as an "honnête homme" what he over-enunciates as a Christian. The enunciature "posture" therefore casts a new light on the dialectical movement at work in Pascal's logic and ontology. This article emphasizes the role played by rhetorics of allusion and disposition in the elaboration of what could be called "postural tropes".

INDEX

Keywords : Pascal, Montaigne, rewriting, allusion, Biblical rhetoric, overenunciation, coenunciation, postural trope.

Mots-clés : Pascal, Montaigne, réécriture, allusion, rhétorique biblique, surénonciation, coénonciation, trope postural. 
AUTEUR

LAURENT SUSINI

Paris-Sorbonne UniversitéSTIH 